\title{
Response to: Comparison of Posterior Lumbar Interbody Fusion and Posterolateral Lumbar Fusion in Monosegmental Vacuum Phenomenon within an Intervertebral Disc
}

\author{
Ki-Chan An, Gyu-Min Kong, Dae-Hyun Park, Jong-Min Baik, Ji-Hong Youn, Woon-Seong Lee \\ Department of Orthopaedic Surgery, Inje University Busan Paik Hospital, Inje University College of Medicine, Busan, Korea
}

Thank you for your queries. I will do my best to answer your questions.

(1) Unfortunately, we did not consider magnetic resonance (MR) findings of patients in this article. Because the primary purpose of this study was comparing of fusion rate about two different fusion modalities, only X-ray findings were retrospectively checked and correlated with surgical indication. MR images of patients were checked prior to surgery as part of the surgical planning. This information was not mentioned in the paper. We will bear your advice in mind in future manuscripts.

(2) Dynamic radiographs, whole spine films and Lspine computed tomography (CT) were obtained for surgical planning and were analyzed for factors including instability, facet joint ossification and calcified disc. To minimize inter- and intra-observer errors, two radiologists independently evaluated the lumbar plane X-rays. If their assessment of the lumbar plane film differed, a CT scan was used as a follow-up procedure.

(3) Indeed, patients with spondylolisthesis and scoliosis were excluded. The case displayed in Fig. 1 (posterior lumbar interbody fusion, PLIF) does show mild spondylolisthesis (grade 1). This is an error caused by the inclusion of an incorrect X-ray. I apologize. In the case displayed in Fig. 2 (posterolateral lumbar fusion, PLF), the patient had no degenerative lumbar scoliosis disease. I think that the lumbar curve is a sciatic curvature adopted to avoid pain, and not true scoliosis.

(4) Unfortunately, I did not consider disc height, sagittal alignment and segmental lordosis. As mentioned above, the primary purpose was comparison of the fusion rate of two different fusion modalities. I will try and consider these factors in future analyses.

(5) Your point concerning bone mineral density is correct. I did not consider this factor.

(6) With respect, there are no errors about blood loss and operation time on page 97. I agree with you concerning their mention otherwise in other articles. However, there were no significant differences between two fusion modalities in my study. I can only report what we found. Furthermore, in our hands the PLIF procedure was longer and the blood loss was greater compared to the PLF procedure. These aspects may be reasons for more operative procedures for PLIF, such as cage insertion, endplate preparation and discectomy.

\section{Conflict of Interest}

No potential conflict of interest relevant to this article was reported.

\footnotetext{
Received July 14, 2015; Accepted July 17, 2016

Corresponding author: Jong-Min Baik

Department of Orthopaedic surgery, Inje University Busan Paik Hospital, Inje University College of Medicine,

75 Bokji-ro, Busanjin-gu, Busan 47392, Korea

Tel: +81-51-890-6114, Fax: +82-51-892-6619, E-mail: bbaik@hanmail.net
} 